\section{$\underset{\substack{\text { hommes } \\ \text { \& migrations }}}{ }$}

\section{Hommes \& migrations}

Revue française de référence sur les dynamiques

migratoires

$1311 \mid 2015$

Femmes et migrations

\title{
La Ligue des droits de l'homme
}

chronique d'un engagement

\section{Françoise Picho-Varin}

\section{(2) OpenEdition \\ 1 Journals}

\section{Édition électronique}

URL : http://journals.openedition.org/hommesmigrations/3349

DOI : 10.4000/hommesmigrations.3349

ISSN : 2262-3353

Éditeur

Musée national de l'histoire de l'immigration

\section{Édition imprimée}

Date de publication : 1 juillet 2015

Pagination : 152-155

ISBN : 978-2-919040-32-2

ISSN : 1142-852X

Référence électronique

Françoise Picho-Varin, "La Ligue des droits de l'homme », Hommes \& migrations [En ligne], 1311 |

2015, mis en ligne le 23 février 2016, consulté le 15 septembre 2020. URL : http://

journals.openedition.org/hommesmigrations/3349 


\section{MÉMOIRES}

\section{LA LIGUE DES DROITS DE L'HOMME ET LA MEEAO} CHRONIQUE D'UN ENGAGEMENT

par FRANÇOISE PICHON-VARIN, Ligue des droits de l'Homme-Paris 12.

$\mathrm{D}$ ifférents événements ont amené la section Paris $12^{\mathrm{e}}$ de la Ligue des droits de l'Homme et du collectif de vigilance Paris $12^{\mathrm{e}}$ pour les droits des étrangers - RESF à se préoccuper du sort de la Maison des étudiants d'Afrique de l'Ouest (MEEAO) et de ses habitants. Ce bâtiment avait servi sous la IVe République (1947-1958) à loger les députés et sénateurs des sept territoires de l'Afrique occidentale française lors des sessions parlementaires.

Dès septembre 1997, la section du $12^{\mathrm{e}}$ arrondissement de la LDH a saisi Jean-François Pernin, alors maire du $12^{\mathrm{e}}$, pour prévenir tout risque d'incident à l'occasion de la fête "Bleu-Blanc-Rouge" organisée par le Front national sur la pelouse de Reuilly. De graves incidents s'étaient produits deux ans auparavant avec l'attaque de la MEEAO et l'agression de plusieurs résidents. Par ailleurs, ayant fait l'objet depuis 2006 de plusieurs rapports des services de sécurité de la préfecture de police et de la Ville, l'immeuble s'est vu frappé en décembre 2009 d'un arrêté interdisant son occupation partielle, puis totale en octobre 2010 pour des raisons de sécurité. Plusieurs résidents qui fréquentaient le conseil de quartier Vallée de Fécamp et ses deux associations (LDH et collectif) leur ont alors demandé conseil et aide.

Deux éléments ont contribué à accroître leur inquiétude : l'engagement par la Ville en 2008 de la procédure dite de "bien sans maître" sur l'im- meuble (aucun des sept États d'Afrique de l'Ouest propriétaires ne s'étant manifesté depuis des années) et en mars 2009 un recensement des résidents réalisé par les services de la Préfecture pour commencer à reloger ceux disposant d'un titre de séjour. Ce recensement restera pour la Préfecture son document de référence tout au long de l'opération, alors que les associations se battront pour qu'elle prenne également en compte, en plus des 93 recensés, la quinzaine de résidents absents ce jour-là de la MEEAO.

\section{L'origine de la mobilisation}

La LDH - Paris 12 ayant appris que la préfecture de police envisageait de procéder à l'évacuation d'un certain nombre de chambres de la MEEAO pouvant concerner une centaine de personnes, elle a immédiatement saisi la maire du $12^{\mathrm{e}}$ pour qu'elle fasse en sorte que cette intervention se déroule dans le respect de la dignité des personnes et de leurs biens. Après lui avoir dit qu'elle ne s'opposerait pas à l'évacuation du fait des risques d'incendie et d'effondrement invoqués, la LDH - Paris $12^{\mathrm{e}}$ lui a demandé d'exiger des autorités compétentes d'être prévenue à l'avance de la date et de l'heure de l'intervention pour que les personnes aient le temps de préparer leurs bagages et de mettre en 
Des membres du collectif de vigilance Paris $12^{\mathrm{e}}$ pour les droits des étrangers - RESF et des résidents de la MEEAO, le 4 septembre 2010, avant de partir pour la manifestation "Face à la xénophobie et à la politique du pilori : Liberté - Egalité - Fraternité".

(c) GiLbert Loeb.

lieu sûr les documents les plus importants et leurs affaires personnelles. Elle a également demandé que les enfants ne soient pas éloignés de leur lieu de scolarité et les adultes de leur lieu de travail. Elle a enfin souhaité que des élus soient présents le jour de l'évacuation.

Plusieurs rencontres informelles s'étaient tenues à partir de juin 2008 dans l'enceinte de l'immeuble avec les résidents de la MEEAO, la LDH Paris 12 et le collectif pour échanger sur la situation présente et à venir de l'immeuble. Malgré l'absence d'interlocuteur représentatif des résidents - deux associations existent et sont souvent en désaccord -, ces échanges ont permis de faire prendre conscience de la nécessité de remédier à la dégradation du bâtiment.

Des courriers ont été envoyés aux ambassades des sept pays africains anciens propriétaires pour leur demander d'intervenir mais sont restés sans réponse (sauf le Sénégal). Aussitôt après la première évacuation du 28 janvier 2010 qui a concerné 69 adultes et 12 enfants, la LDH - Paris 12, le Collectif de vigilance et les représentants de la MEEAO ont demandé à la préfecture de Paris la mise en place de réunions régulières. La première revendication fut d'avoir un interlocuteur unique qui traite à la fois la question de l'hébergement et du relogement mais aussi celle de la régularisation avec un traitement spécifique de la préfecture de police sur les dossiers MEEAO.

\section{Le combat avec les résidents pour les relogements}

La LDH - Paris 12 a organisé des rencontres hebdomadaires avec les résidents évacués et ceux habitant encore l'immeuble jusqu'à la deuxième évacuation du 20 octobre 2010, touchant 77 adultes et 19 enfants. Ces réunions ont permis de suivre la situation de chacun et aux familles hébergées en hôtel de faire part des difficultés rencontrées : 


\section{MÉMOIRES}

impossibilité de cuisiner, éloignement par rapport aux écoles et aux lieux de travail, annonce répétée de la fin de leur hébergement et de la mise à disposition de garde-meubles. Ces demandes étaient immédiatement transmises par les associations et traitées lors des réunions avec la préfecture de Paris.

Les rencontres avec les ex-résidents sont ensuite passées à un rythme bimensuel. Au fur et à mesure des relogements, il est apparu de plus en plus difficile de faire venir les gens à ces réunions. On s'est alors appuyé sur plusieurs résidents très impliqués depuis le début qui se sont répartis les personnes à prévenir et sont devenus les porteparoles des familles et ont de ce fait été associés aux réunions avec les partenaires publics.

Ces réunions ont également permis de décider d'actions à mettre en place pour maintenir la mobilisation. Parmi les actions proposées et réalisées, on peut mentionner la confection par les résidents d'une banderole rappelant qu'ils n'étaient pas des squatteurs et que tous avaient droit à un relogement : "Relogement, Régularisation, Tenez vos promesses". Cette banderole a été accrochée en mai 2010 sur le fronton de l'immeuble. Le 8 juin 2010, entre la première et la deuxième évacuation, un dîner festif organisé dans la MEEAO par les représentants des sept pays africains à l'intention de l'ensemble des résidents et des habitants du quartier a rassemblé plus de soixante personnes. En novembre 2010, peu de temps après la deuxième évacuation, un collectif d'artistes du quartier a réalisé des pochoirs sur les fenêtres murées de la MEEAO reproduisant des extraits de poèmes et des textes de Senghor, de Césaire et d'autres poètes africains, ainsi que des photos des résidents prises par plusieurs jeunes photographes impliqués dans cette lutte depuis le début. Une frise des prénoms des personnes ayant habité l'immeuble a été peinte sur les murs et les fenêtres murées. En janvier 2012, une exposition a été présentée pendant plusieurs semaines dans la mairie $d u 12^{\mathrm{e}}$, arrondissement de Paris retraçant avec des photos, reportages et document, l'histoire de la MEEAO, la vie de ses occupants, les évacuations et les combats qui les ont accompagnés.

\section{Les négociations avec la préfecture et la Ville de Paris}

La préfecture de Paris a répondu à la demande de réunions régulières de la LDH - Paris 12 auxquelles ont été associés les représentants des résidents. Six réunions se sont tenues avec le cabinet du préfet afin de suivre les hébergements des familles relogées provisoirement dans les hôtels. Le préfet de région, préfet de Paris, a mandaté le groupement d'intérêt public Habitat et interventions sociales (GIP HIS) pour que ses travailleurs sociaux contactent les personnes hébergées en hôtel, notamment celles avec un titre de séjour, et leur fassent des propositions de relogement. On a sollicité le GIP pour qu'il propose également des relogements aux familles dont un seul membre du couple détenait un titre de séjour ou l'avait depuis peu de temps, malheureusement sans succès.

Il a fallu négocier à de nombreuses reprises la prolongation des hébergements en hôtel, prévus au départ pour un mois. Les résidents, notamment ceux sans titre de séjour, alertaient les associations dès qu'un hôtelier leur signifiait la fin de leur prise en charge, alors qu'ils n'avaient aucune solution de relogement. Les associations appelaient en urgence la Préfecture. Les hébergements ont pu être prolongés jusqu'à fin mai 2010. Un rassemblement fut organisé par les résidents et les associations le 26 février 2010 devant la Préfecture au métro Sully-Morland et plusieurs communiqués ont été publiés, rappelant que l'État devenu propriétaire de l'immeuble était tenu de 
reloger "les occupants de bonne foi", qu'ils aient ou non un titre de séjour.

Les résidents et les associations ont souhaité rencontrer également la Ville de Paris, bien que celleci ait rappelé ne pas être légalement concernée par le cas de la MEEAO, puisque l'État en était le propriétaire. Elle a néanmoins pris en charge sur son contingent le relogement de 11 ménages, y compris les couples dont un seul membre disposait d'un titre de séjour.

À l'occasion d'une émission sur Radio libertaire consacrée à la MEEAO, le dernier recensement des relogements effectué par la LDH - Paris 12 en juillet 2011 fait état de 55 ménages (célibataires et familles) relogés, une dizaine hébergés en hôtel social, souvent des couples dont un seul membre est en situation régulière, une trentaine dont la prise en charge en hôtel a pris fin, les obligeant à trouver une solution par leurs propres moyens. Cette catégorie concerne de nombreux célibataires sans titre de séjour ou régularisés après l'évacuation et qui ont dû trouver une solution dans le parc privé en attendant que leur demande de logement social aboutisse.

La régularisation des résidents dépourvus de titre de séjour constitue le deuxième volet de la mobilisation. Bien qu'étant en France depuis de nombreuses années avec des preuves de présence depuis huit, dix ans voire plus, un certain nombre de résidents n'avaient jamais déposé de demande. Parmi la soixantaine ayant sollicité de l'aide pour constituer leur dossier de demande de régularisation, une vingtaine d'entre eux, des célibataires ou des familles, étaient bien connus du collectif depuis plusieurs années. Ils avaient participé aux parrainages républicains de personnes sans papiers organisés depuis 2006 par le collectif avec la mairie du $12^{\mathrm{e}}$ arrondissement de Paris. Avec l'aide des parrains-marraines, le collectif a consacré de nombreuses heures lors des permanences du samedi matin à préparer les dossiers. La liste était donnée au fur et à mesure à notre interlocuteur de la Préfecture qui se chargeait de la transmettre au préfet de police pour qu'il convoque rapidement les personnes. Chacune était accompagnée pour son rendez-vous par le Collectif de vigilance. Ce sont trente à quarante personnes au minimum qui ont obtenu leur titre de séjour.

\section{Les enseignements de la lutte}

Le combat autour de la MEEAO reste pour les militants de la LDH - Paris 12 et du Collectif de vigilance un épisode à la fois éprouvant et passionnant qui les a conduits à ressentir la violence qu'a représentée, pour les résidents, l'arrachement à ce qui fut pendant des années leur lieu de vie et la séparation avec les amis alentour, mais aussi à constater la solidarité qui s'est tissée avec eux au fil des mois et des années de lutte.

Déception et colère vis-à-vis d'un État devenu propriétaire de l'immeuble et qui n'a jamais voulu reconnaitre son obligation de relogement vis-à-vis de résidents "occupants de bonne foi", quelle que soit leur situation administrative, ce qui obligea les associations à négocier au cas par cas et sans relâche aussi bien l'hébergement et le relogement que la régularisation.

Un demi-succès par rapport aux revendications portées par les associations et les représentants des résidents dès le début des rencontres avec la Préfecture, notamment que ce bâtiment garde sa vocation d'habitat social et que la mémoire du lieu soit conservée. Ces deux revendications sont en passe d'aboutir puisque la gestion de l'immeuble a été confiée à un bailleur social et que des réflexions sont en cours avec lui et les artistes qui s'étaient mobilisés en 2010 pour choisir comment perpétuer au mieux la mémoire de ce lieu chargé d'histoire. 